\title{
Aging affects the balance between goal-guided and habitual spatial attention
}

\author{
Emily L. Twedell ${ }^{1}$ - Wilma Koutstaal ${ }^{1}$ • Yuhong V. Jiang ${ }^{1}$
}

Published online: 19 December 2016

(C) Psychonomic Society, Inc. 2016

\begin{abstract}
Visual clutter imposes significant challenges to older adults in everyday tasks and often calls on selective processing of relevant information. Previous research has shown that both visual search habits and task goals influence older adults' allocation of spatial attention, but has not examined the relative impact of these two sources of attention when they compete. To examine how aging affects the balance between goal-driven and habitual attention, and to inform our understanding of different attentional subsystems, we tested young and older adults in an adapted visual search task involving a display laid flat on a desk. To induce habitual attention, unbeknownst to participants, the target was more often placed in one quadrant than in the others. All participants rapidly acquired habitual attention toward the highprobability quadrant. We then informed participants where the high-probability quadrant was and instructed them to search that screen location first- but pitted their habit-based, viewer-centered search against this instruction by requiring participants to change their physical position relative to the desk. Both groups prioritized search in the instructed location, but this effect was stronger in young adults than in older adults. In contrast, age did not influence viewer-centered search habits: the two groups showed similar attentional preference for the visual field where the target was most often found before. Aging disrupted goal-guided but not habitual attention. Product, work, and home design for people of all ages - but especially for older individuals - should take into
\end{abstract}

Yuhong V. Jiang

jiang166@umn.edu

1 Department of Psychology, University of Minnesota, 75 East River Road, S251 Elliott Hall, Minneapolis, MN 55455, USA account the strong viewer-centered nature of habitual attention.

Keywords Aging · Visual attention · Implicit learning · Spatial reference frame

Most scenes contain visual clutter, where objects or features relevant to task goals are surrounded by irrelevant visual information. Such visual clutter can impose challenges for everyday activities, such as driving and reading, and is particularly detrimental for older adults. When navigating a website, for instance, older adults perform more poorly than young adults when the relevant information is embedded among visually similar distractors (Grahame, Laberge, \& Scialfa, 2004). To deal with clutter, people need to selectively process information from important locations. Visual attention in both older and young adults is influenced by prior learning and habits. For example, when relevant text is placed on the left side of a website, a region that left-to-right readers habitually search first, effects of visual clutter are attenuated in older adults (Grahame et al., 2004; see also McCarley, Yamani, Kramer, \& Mounts, 2012). Older and young adults can also rely on top-down goals to prioritize processing, such as when instructed to attend to the right side of a screen (Olk \& Kingstone, 2015). Because task goals sometimes conflict with acquired habits, performance reflects the combined effects of goals and habits. To date, few studies have investigated the relative contribution of goal-guided versus habit-guided attention to older adults' visual search performance. When in conflict, does one source of attention dominate over the other? How does aging influence the balance between goal-driven and habit-guided 
attention, and how might aging inform our understanding of different subsystems of spatial attention?

One recent study suggests that, in older adults, the balance may be tipped toward habit-based rather than goal-guided search (Jiang, Koutstaal, \& Twedell, 2016). Young and older adults searched for a $\mathrm{T}$ target among $\mathrm{L}$ distractors. Unbeknownst to the participants, the T was more often placed in one visual quadrant in the first part of the experiment. Even though participants were unaware of the T's location probability, they were faster finding the $\mathrm{T}$ in the high-probability quadrant than in the low-probability quadrants. Once learned, the search habit persisted for several hundred trials, even after the target location was changed to being random. Compared with young adults, older adults' response times were slower, and increasing the number of distractors differentially impaired performance of older adults. However, older adults showed robust location probability learning, searching fastest in the high-probability quadrant. This finding suggests that habit-driven attention may be preserved in older adults. However, Jiang et al. (2016) did not directly manipulate goal-driven spatial attention; thus their results do not address how habitual and goal-driven attention combine to influence older adults' performance.

Research with young adults suggests that, when in conflict, goal-driven attention coexists with, or overrides, habitual attention (Jiang, Swallow, \& Rosenbaum, 2013). Participants first acquired a search habit toward one quadrant through location probability learning. Next, they were told to prioritize, on a trial-by-trial basis, locations indicated by a central arrow. In this second phase, performance was determined primarily by the validity of the arrow: Reaction time (RT) was faster when the target appeared in the quadrant cued by the arrow than when it appeared elsewhere. The previously learned search habit had little influence on RT.

However, goal-driven attention does not always override habitual attention in young adults. Taking advantage of the observation that habitual attention is viewer centered, researchers introduced a competition between viewer-centered, habitual attention and environment-centered, goal-driven attention (Jiang, Swallow, \& Sun, 2014). Participants performed visual search on a display laid flat on a desk. Following location probability training, participants moved to an adjacent edge of the desk, thereby changing their viewpoint by $90^{\circ}$. In the absence of additional instructions, the learned habit persisted in a viewercentered manner. Participants prioritized the same visual field as the previously high-probability quadrant, but not the same screen locations. When a conflicting goal-driven cue was provided-for instance, when participants were instructed to prioritize the same screen location as where the target was most often found-young adults were influenced by both task instructions and their learned habit. They continued to respond more quickly if the target appeared in the same visual field as the previously highprobability quadrant (viewer centered), as well as in the instructed environment-centered quadrant. This finding suggests that, for young adults, goal-driven attention can coexist with conflicting search habits.

This study examines how conflicting sources of spatial attention influence visual search in older adults. We adopted the experimental paradigm used in Jiang et al. (2014) that pits viewer-centered habitual attention against environment-centered goal-driven attention. Two lines of previous research suggest that, relative to young adults, older adults are more likely to rely on search habits than task instructions. First, as shown in Jiang et al. (2016), location probability learning is preserved in older adults, even though their search is less efficient relative to young adults. Second, research on age differences in the use of spatial reference frames suggests that aging has a more detrimental effect on environment-centered coding than viewer-centered coding. For example, participants in one study were instructed to code an object's location in a specific reference frame, either environment-centered or viewer-centered (Montefinese, Sulpizio, Galati, \& Committeri, 2015). Participants then moved to another location and were asked to report the object's location according to the instructed frame of reference. Older adults were impaired in reporting the object's location in an environment-centered reference frame, but were intact in reporting the object's location in a viewer-centered reference frame (see also Lemay, Bertram, \& Stelmach, 2004; Lithfous, Dufour, Blanc, \& Després, 2014, for analogous results). However, in Montefinese et al.'s study, the use of different reference frames was explicitly required by the task. It is unclear whether older adults also rely strongly on viewer-centered representations when a viewer-centered representation is acquired incidentally.

In contrast to the results from Jiang et al. (2016) suggesting that aging may tip the balance of attention from goal-driven to habitual attention, neuroimaging studies have shown agerelated decline in the function of a brain region important for habit formation - namely the basal ganglia (for review, see Howard \& Howard, 2013). Tasks that depend on basal ganglia function, such as learning a probabilistic sequence, are performed more poorly by older than young adults (Howard, Howard, Dennis, Yankovich, \& Vaidya, 2004). These findings could imply that even though older adults can acquire habitual attention when no conflicting task goals exist, in the presence of competition between habit-driven and goal-driven attention, any age-related decline in the habit system will preferentially facilitate goal-driven attention.

This study directly tests these two conflicting predictions. We examine whether, when placed in competition, the habitbased or goal-driven attention subsystem predominates in guiding the visual search performance of older adults. 


\section{Method}

Participants Participants were 24 young adults (18-30 years) and 24 older adults (60-80 years). All participants had normal or corrected-to-normal vision and hearing, normal color vision, were native English speakers, and self-reported no history of serious health problems such as psychiatric or neurological disorders, heart condition, hypertension, uncontrolled diabetes, or chemical dependence. Table 1 lists demographic information.

Equipment The visual search task was administered individually in a room lit by a 25 -watt lamp. The testing desk was at a corner of the rectangular room. Participants sat at one edge of the desk, looking down at a 19-inch LCD monitor placed flat on the desk. The room furniture was readily visible. Viewing distance was approximately $40 \mathrm{~cm}$ to the center of the screen.

Materials Participants conducted visual search for a letter T. Each display contained one $\mathrm{T}$ and several Ls, presented in randomly selected locations within an invisible $10 \times 10$ matrix $\left(32^{\circ} \times 32^{\circ}\right)$. The background was black. The number of Ls was randomly determined to be seven, 11 , or 15 . The varying number of distractors allowed us to assess effects of incidental learning on visual search efficiency. An equal number of items appeared in each quadrant. An outline frame enclosed the search space. One thick edge of the frame was blue $\left(39^{\circ} \times\right.$ $\left.2.3^{\circ}\right)$; it remained in the same external space throughout the experiment. The other three thin edges $\left(39^{\circ} \times 0.2^{\circ}\right)$ were white (see Fig. 1). Each item $\left(2^{\circ} \times 2^{\circ}\right)$ was oriented in a random direction and had a red or green tint. The RGB values were [250 188 188] for red and [188 250 188] for green. Participants pressed $r$ for a red T or $g$ for a green T.

Design and procedure Participants performed 17 practice trials using random target locations. Next, participants completed the training, recognition, and testing phases.

Training The first eight blocks (36 trials each) constituted the training phase. Without informing participants, we placed the $\mathrm{T}$ in one quadrant on $50 \%$ of the trials, and in each of the other quadrants $16.7 \%$ of the time. The high-probability quadrant was counterbalanced across participants and remained consistent for a given participant.

Recognition After training, an experimenter administered a surprise recognition test while participants remained at the training position. Participants were asked whether they thought the target was equally likely to appear anywhere on the screen or whether it was more often found in some locations than others. Regardless of their response, participants were next informed that the target was more often located in one quadrant. They were asked to click on the quadrant where
Table 1 Participant characteristics. Standard deviation is in parenthesis

\begin{tabular}{llll}
\hline & Older & Young & Difference \\
\hline Age & $68(5)$ & $20(3)$ & $p<.001$ \\
Years of education & $17(2)$ & $15(3)$ & $p<.001$ \\
Gender (F:M) & $17: 7$ & $17: 7$ & $p>.50$ \\
Shipley Nonverbal IQ & $116(12)$ & $107(10)$ & $p<.01$ \\
Shipley Verbal IQ & $112(9)$ & $108(10)$ & $p>.20$ \\
Shipley Composite IQ & $116(7)$ & $110(10)$ & $p<.05$ \\
\hline
\end{tabular}

the target was most often found. Participants then rated their confidence on a 4-point scale ( $1=$ guessing; $4=$ certain $)$.

Testing Following the recognition responses, participants received information about where the high-probability quadrant was located. A blue outline frame enclosed that quadrant. The experimenter explained that this quadrant was where the target was most often found. Next, participants were asked to move their seat to an adjacent side of the desk $\left(90^{\circ}\right.$ counterclockwise) and to complete the experiment while sitting there. The display and the blue frame remained in view and were unchanged. After the participants had moved, the experimenter pointed at the blue frame and indicated that, for the rest of the experiment, participants should search that region first. No participants inquired about the rationale behind this instruction. Participants completed four blocks (36 trials each). Crucially, the target's location was rendered random during this phase (25\% in each quadrant).

Standardized cognitive test To ensure that older adults did not have a general reduction in cognitive function relative to our sample of young adults, we obtained scores for verbal, nonverbal, and composite intelligent quotient (IQ) using subtests of vocabulary and abstract reasoning from the Shipley Institute of Living Scale-2 (Shipley-2; www. creativeorgdesign.com).

\section{Results}

Similar to previous findings (Salthouse, 2012), older adults had higher IQ and more years of education than young adults (see Table 1). This helps rule out IQ differences as a potential explanation for any impairment in older adults in the visualspatial attention task.

\section{Accuracy and RT adjustment}

Both groups were highly accurate, making less than $3 \%$ of errors in the visual search task. However, similar to a previous study (McLaughlin \& Murtha, 2010), older adults were slightly more accurate (99\%) than were young adults (97\%), $p<$ 
a

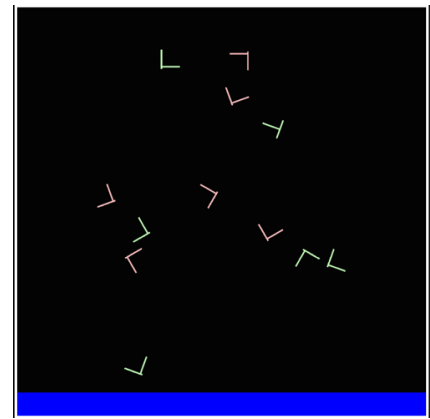

Find T b

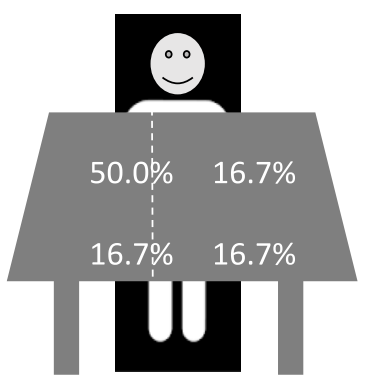

Training: Unequal
C

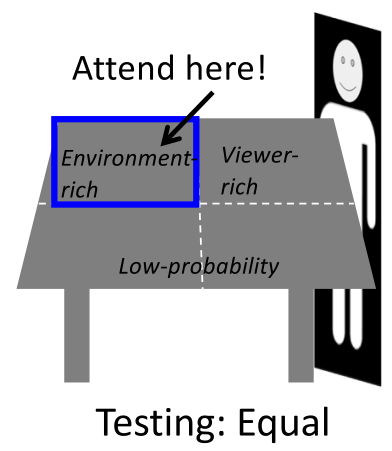

Fig. 1 Experimental setup. a A sample search display placed flat on the desk. Participants reported the color of the letter T. b Training phase. The target occurred 3 times more frequently in one quadrant than in any of the other quadrants. $\mathbf{c}$ Testing phase. Participants changed their viewpoint by $90^{\circ}$. The target was equally likely to appear in any quadrant. Participants were instructed to prioritize the same screen location as the previously trained high-probability quadrant (i.e., the "environment-rich" quadrant)
.05. Age group did not interact with other experimental factors. Given that accuracy was high and it did not interact with age group, we focused on reaction time (RT) as the main dependent measure, excluding incorrect trials.

\section{Training phase}

Figure 2a shows mean RT in the training phase. Older adults were slower than young adults. This baseline difference could mask other experimental effects. Following a method designed to address group differences in baseline response latency (Faust, Balota, Spieler, \& Ferraro, 1999), we converted trial RT into $z$ scores. This conversion was performed for each individual $(z=$ [trial RT - individual mean RT] / individual standard deviation) and for training and testing phases separately. All statistical analyses were conducted on the $z$ scores (see Fig. 2b).

We entered the target's quadrant condition (high or low probability) and block as within-subject factors, and agegroup as a between-subject factor in an ANOVA. This analysis showed significant main effects of quadrant condition and block and no other effects. Participants were faster finding the target in the high-probability than the low-probability quadrants, $F(1,46)=108.92, p<.001, \eta_{\mathrm{p}}{ }^{2}=.70$. This effect was comparable between young and older adults, resulting in a lack of interaction between age-group and target quadrant, $F(1,46)=2.92, p>.09$. RT became faster in later blocks than earlier ones, $F(7,322)=17.70, p<.001, \eta_{\mathrm{p}}{ }^{2}=.28$. None of the other interaction effects were significant, all $p \mathrm{~s}>.50$. Thus, older adults had a preserved ability to form a habitual attentional preference for the high-probability locations.

In the analysis reported above, we combined data across all set sizes. In a further analysis, we examined how incidental learning affected search across varying set sizes. As shown in Table 2, participants were slower when more items were on the display. This set-size effect was greater in the low- probability quadrants than the high-probability quadrant, yielding a significant interaction between target quadrant and set size, $F(2,92)=7.95, p<.001, \eta_{\mathrm{p}}{ }^{2}=.15$. This effect was found in both young and older adults and hence did not interact with age-group, $F<1$. This finding indicates that incidental learning enhanced the efficiency of visual search.

\section{Recognition}

About half of the participants ( $54 \%$ of older adults and $54 \%$ of young adults) said the target was more often in some locations than others. When given the forced-choice about the quadrant most often containing the target, $71 \%$ of older and $63 \%$ of young adults correctly identified the high-probability quadrant, which was significantly above chance, $\chi^{2}(1) \mathrm{s}>18.00$, $p>.001$. Both groups expressed some confidence in their recognition choice. On a 4-point scale, the median confidence rating was 2 for both young and older adults, Mann-Whitney test $z$ score $=1.13, p>.20$.

To examine the possible impact of explicit awareness on location probability learning, we classified participants as aware or unaware. The aware participants were those who correctly determined that the target's location was uneven and accurately identified the high-probability quadrant (42\% of older and $46 \%$ of young adults). Other participants were the "unaware" participants. Next, we calculated RT saving for each participant as the difference in $z$ score between the high- and low-probability quadrants. An ANOVA using agegroup and awareness status as between-subject factors showed no effects of age, awareness status, or their interaction, all $p \mathrm{~s}>$ .10. On average, the aware participants $(N=21)$ showed a saving of $z=0.41$, and the unaware participants $(N=27)$ showed a saving of $z=0.37$, both of which were significantly above zero, $p<.001$. Thus, awareness was not necessary for acquiring a habitual shift of attention. 
a
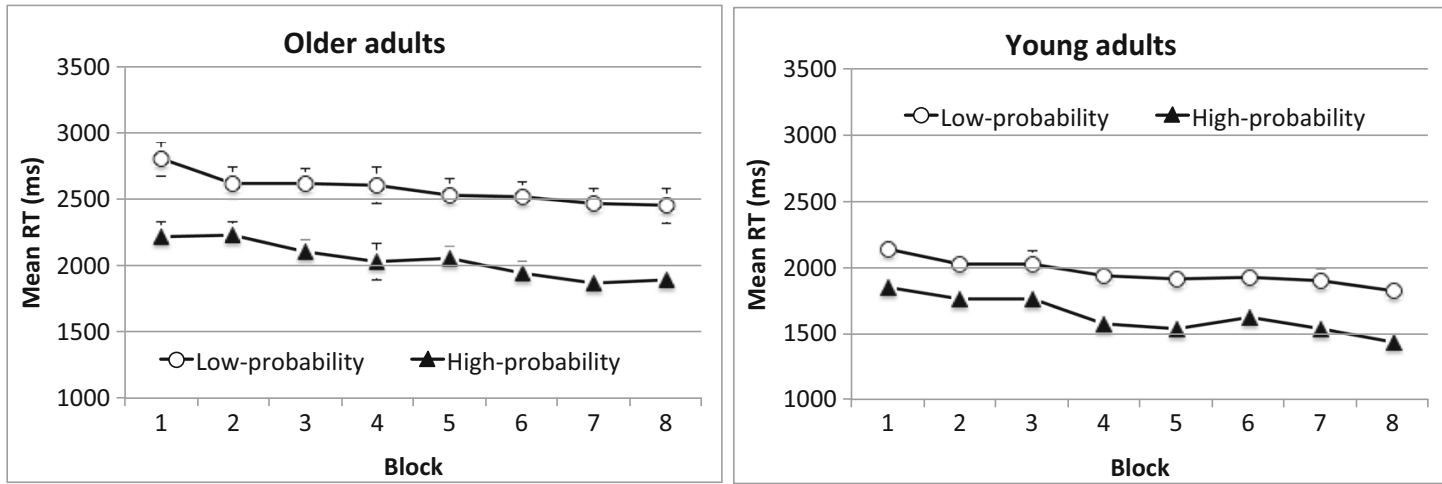

b
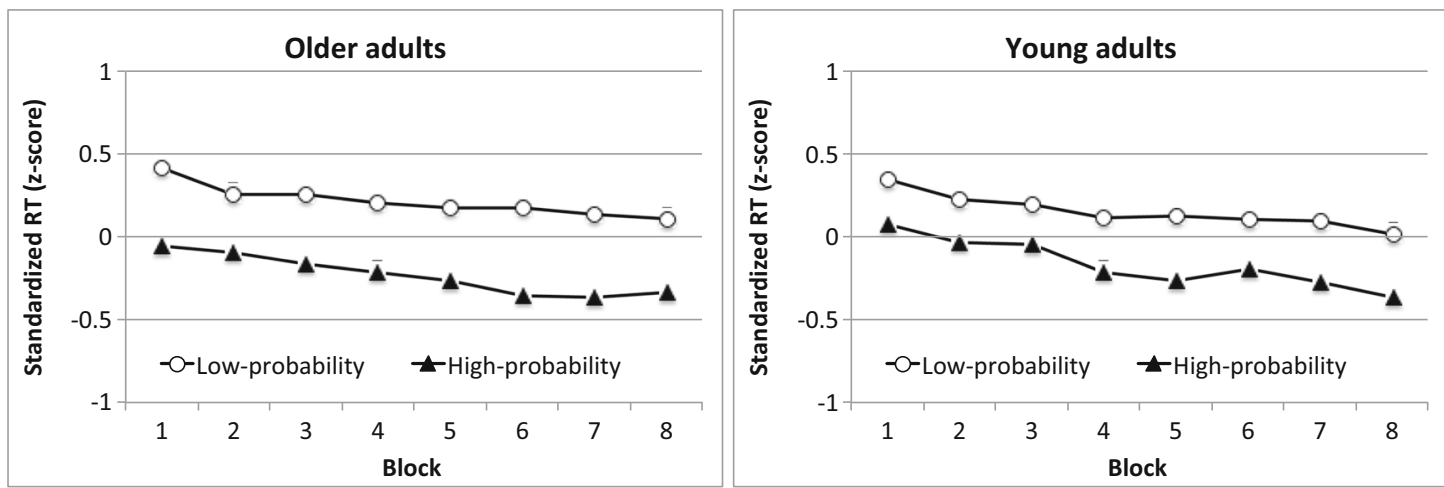

Fig. 2 Results from the training phase. a Mean RT. b Standardized RT in $z$ scores. Error bars show $\pm 1 S E$ of the mean

\section{Testing phase}

Next, we examined how the learned attentional habit affected subsequent search. In the testing phase the target appeared in each quadrant $25 \%$ of the time. In addition, participants changed their viewpoint by $90^{\circ}$. Because participants were instructed to prioritize the same screen location as the previous high-probability location, successful allocation of attention based on the instruction should yield faster RT in that "environment-rich" quadrant than in the other quadrants. As shown in Fig. 3, this was the case.

An ANOVA including target quadrant (low probability, environment rich, or viewer rich) and age group showed that RT differed significantly across the quadrant conditions, $F(2$, $92)=59.30, p<.001, \eta_{\mathrm{p}}{ }^{2}=.56$. The interaction between condition and age group was significant, $F(2,92)=6.50, p$ $<.002, \eta_{\mathrm{p}}^{2}=.12$.

To understand this quadrant-by-age interaction, we performed two ANOVAs. (All statistical significance reported here remained significant with Bonferroni correction for multiple comparisons.) The first analysis examined how well the two groups used task instructions to prioritize the environment-rich quadrant. An ANOVA including target quadrant (low probability, environment rich) and age group revealed both a significant main effect of target quadrant, $F(1$, 46) $=118.18, p<.001, \eta_{\mathrm{p}}{ }^{2}=.72$, and a significant quadrant by group interaction, $F(1,46)=12.02, p<.001, \eta_{\mathrm{p}}{ }^{2}=.21$. Instructions sped up RT by $0.78 z$ score units in young adults. This declined to 0.40 units in older adults. Although both age groups were significantly above zero in one-sample $t$ tests $(p<$

Table 2 Standardized RT ( $z$ score) for different set sizes. Standard deviation is in parenthesis
Low-probability quadrants
High-probability quadrant

\begin{tabular}{lllllll}
\hline Set size & 8 & 12 & 16 & 8 & 12 & 16 \\
Older & $-0.14(0.03)$ & $0.22(0.04)$ & $0.62(0.05)$ & $-0.48(0.03)$ & $-0.20(0.03)$ & $0.08(0.05)$ \\
Young & $-0.24(0.03)$ & $0.16(0.04)$ & $0.56(0.05)$ & $-0.50(0.03)$ & $-0.15(0.03)$ & $0.19(0.05)$ \\
\hline
\end{tabular}


a

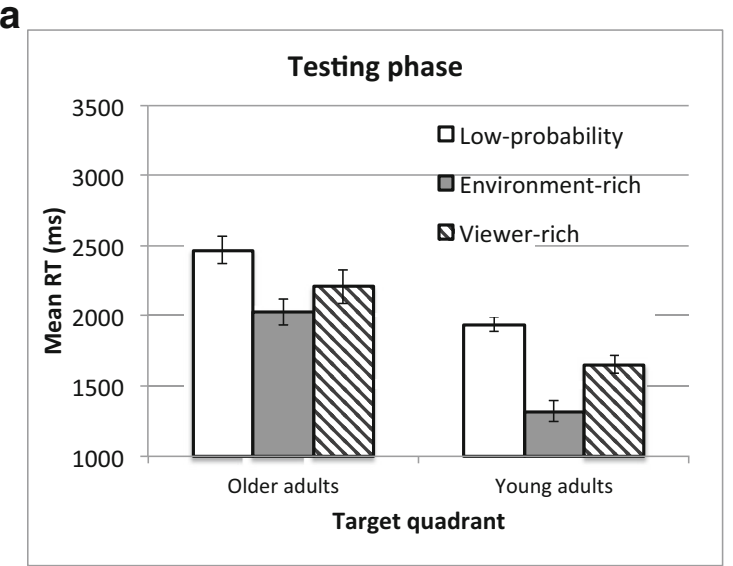

b

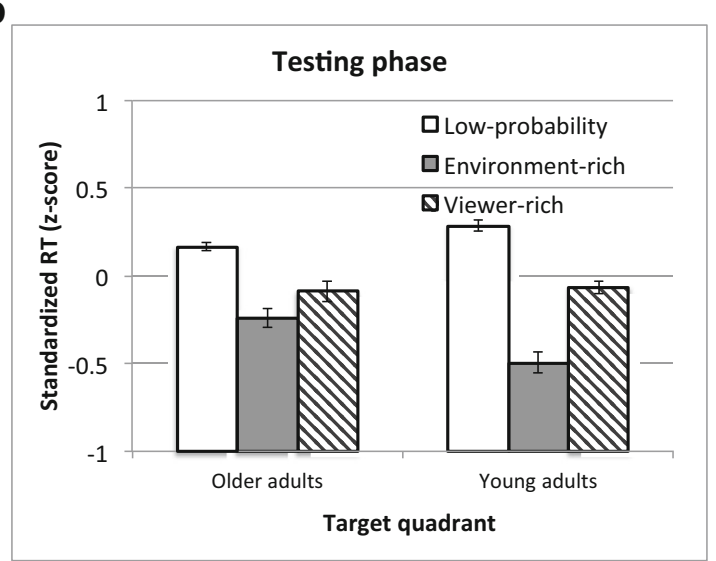

Fig. 3 Results from the testing phase during which the target was equally likely to appear in each quadrant, but participants were instructed to prioritize the environment-centered rich quadrant. Error bars show $\pm 1 S E$ of the mean. a Mean RT. b Standardized RT in $z$ scores

.001), older adults were less able to use task instructions to prioritize the environment-rich quadrant than were young adults.

In the second analysis, we tested the degree to which the two groups prioritized the viewer-rich quadrant. An ANOVA including target quadrant (low probability, viewer rich) and age group showed only a significant main effect of target quadrant, $F(1,46)=44.05, p<.001, \eta_{\mathrm{p}}^{2}=.49$, without a quadrant by age interaction, $F(1,46)=1.14, p>.25$. This viewer-based attentional habit was found even though, at the time of testing, the target's location was random. If anything, the task instruction provided an incentive to attend away from the viewer-rich quadrant. Nonetheless, participants were faster finding the target in the viewer-rich quadrant, an effect comparable in strength between young and older adults.

\section{Discussion}

This study demonstrates that older and young adults rely on two mechanisms to allocate attention: top-down goals and search habits. When set in competition, both mechanisms contribute to search performance. First, explicit task instructions mobilize the goal-driven mechanism. It can be directed toward an external environmentally coded region of space even after a change in viewpoint. Furthermore, the task instruction does not need to be valid. During the testing phase, the target was no more likely to appear in the instructed quadrant than in the other quadrants. This did not prevent participants from using the instructions. Second, incidental learning of the target's location probability induced a search habit toward the highprobability locations. This habit persisted even when the target's location became random, and when participants' viewpoint had changed $90^{\circ}$.

Both older and young adults evidenced this reliance on topdown goals and habit-based search in this study. However, aging affected the relative contribution of the two mechanisms to spatial attention. Compared with young adults, older adults were less effective in intentionally following the instructions. The RT advantage in the instructed, environment-centered quadrant was about half as strong in older adults as in young adults. In contrast, habitual attention was comparable between the two age groups, suggesting that, in this paradigm, agerelated impairments in basal ganglia function did not substantially interfere with habit-based visual search learning. Indeed, for both older and young adults, explicit instructions to prioritize another region were insufficient to eliminate a search habit toward the previously high-probability quadrant, as defined in the viewer-centered space.

Data presented here join an expanding set of findings suggesting that goal-driven and habitual attention may reflect different subsystems of spatial attention. Our study showed that these two types of attention are differentially affected by aging. Other findings have shown a similar dissociation using working memory load: goal-driven attention is adversely affected by additional working memory demands, but habitual attention is not (Won \& Jiang, 2014). Finally, whereas goaldriven attention can be coded independent of viewpoint, habitual attention is strongly viewer centered. These findings support the dual-system view of visuospatial attention (Jiang, Swallow, \& Capistrano, 2013). This view links goaldriven and habitual attention to the multiple subsystems of memory account (Squire \& Wixted, 2011). Goal-driven attention relies on declarative memory, such as knowledge about where to allocate attention. Habitual attention reflects a change in the procedural component of attention, such as how to shift attention. Each time the target is successfully found, the vector of attentional shift that lands on the target is reinforced.

What is the nature of this habit-based attention subsystem? Is it instantiated in the motoric system, such as the tendency to move one's eyes in a specific direction? Although our study 
does not address this question, previous studies showed that frequently shifting one's eyes to a quadrant was neither necessary (Won \& Jiang, 2014) nor sufficient (Jiang et al., 2013) for developing location probability cuing. Habitual attention is rooted in the premotor cognitive processing level, rather than the motor level. That is, the search habit is enhanced via attentional guidance that is independent of ocular movementperhaps also related to why habit-based visual search learning proves to be intact in older adults despite any possible agerelated impairments in basal ganglia function.

Our study suggests that older adults are relatively intact in deploying spatial attention through incidental habit-based learning. This finding has practical implications. First, training older adults to develop good search habits may help compensate for a decline in goal-driven attention. Such training should take into account the strong viewer-centered component of habitual attention. For example, consistent placement of important objects or information at home, in shopping centers, and on product labels can facilitate the acquisition and use of an adaptive search habit. Changes in viewpoint should be minimized by reducing the number of entrances to a facility or by automatically reorienting stimuli according to the viewers' perspective. Second, owing to the strong persistence of a learned habit and a decline in goal-driven attention, older adults will require different training interventions to compensate for maladaptive search habits. Corrective training should focus on extensive practice to acquire a new and adaptive search habit, in conjunction with explicit instructions. In this regard, maintaining consistencies in spatial designs across different environments may be particularly important.

Acknowledgments This study was supported by the Engdahl Family Research Fund. We thank participants for volunteering in our study, and the Osher Lifelong Learning Institute and the University of Minnesota Retiree Volunteer's Center for help with recruiting participants. We thank Douglas Addleman and Deborah Tan for comments on the manuscript.

\section{References}

Faust, M. E., Balota, D. A., Spieler, D. H., \& Ferraro, F. R. (1999). Individual differences in information-processing rate and amount: Implications for group differences in response latency. Psychological Bulletin, 125(6), 777-799. doi:10.1037//00332909.125.6.777
Grahame, M., Laberge, J., \& Scialfa, C. T. (2004). Age differences in search of web pages: The effects of link size, link number, and clutter. Human Factors, 46, 385-398. doi:10.1518/hfes.46.3.385.3808

Howard, J. H., \& Howard, D. V. (2013). Aging mind and brain: Is implicit learning spared in healthy aging? Frontiers in Psychology, 4, 817. doi:10.3389/fpsyg.2013.00817

Howard, J. H., Howard, D. V., Dennis, N. A., Yankovich, H., \& Vaidya, C. J. (2004). Implicit spatial contextual learning in healthy aging. Neuropsychology, 18(1), 124-134. doi:10.1037/08944105.18.1.124

Jiang, Y. V., Koutstaal, W., \& Twedell, E. L. (2016). Habitual attention in older and young adults. Psychology \& Aging, 31, 970-980. doi:10.1037 /pag0000139

Jiang, Y. V., Swallow, K. M., \& Capistrano, C. G. (2013). Visual search and location probability learning from variable perspectives. Journal of Vision, 13(6), 13. doi:10.1167/13.6.13

Jiang, Y. V., Swallow, K. M., \& Rosenbaum, G. M. (2013). Guidance of spatial attention by incidental learning and endogenous cuing. Journal of Experimental Psychology: Human Perception and Performance, 39(1), 285-297. doi:10.1037/a0028022

Jiang, Y. V., Swallow, K. M., \& Sun, L. (2014). Egocentric coding of space for incidentally learned attention: Effects of scene context and task instructions. Journal of Experimental Psychology: Learning, Memory, \& Cognition, 40(1), 233-250. doi:10.1037/a0033870

Lemay, M., Bertram, C. P., \& Stelmach, G. E. (2004). Pointing to an allocentric and egocentric remembered target in younger and older adults. Experimental Aging Research, 30(4), 391-406. doi:10.1080/03610730490484443

Lithfous, S., Dufour, A., Blanc, F., \& Després, O. (2014). Allocentric but not egocentric orientation is impaired during normal aging: An ERP study. Neuropsychology, 28(5), 761-771. doi:10.1037/neu0000084

McCarley, J. S., Yamani, J. S., Kramer, A. F., \& Mounts, J. R. W. (2012). Age, clutter, and competitive selection. Psychology and Aging, 27, 616-626. doi:10.1037/a0026705

McLaughlin, P. M., \& Murtha, S. J. E. (2010). The effects of age and exogenous support on visual search performance. Experimental Aging Research, 36(3), 325-345. doi:10.1080/0361073X.2010.484752

Montefinese, M., Sulpizio, V., Galati, G., \& Committeri, G. (2015). Agerelated effects on spatial memory across viewpoint changes relative to different reference frames. Psychological Research, 79(4), 687697. doi:10.1007/s00426-014-0598-9

Olk, B., \& Kingstone, A. (2015). Attention and ageing: Measuring effects of involuntary and voluntary orienting in isolation and in combination. British Journal of Psychology, 106(2), 235252. doi:10.1111/bjop.12082

Salthouse, T. (2012). Consequences of age-related cognitive declines. Annual Review of Psychology, 63, 201-226. doi:10.1146/annurevpsych-120710-100328

Squire, L. R., \& Wixted, J. T. (2011). The cognitive neuroscience of human memory since H.M. Annual Review of Neuroscience, 34, 259-288. doi:10.1146/annurev-neuro-061010-113720

Won, B.-Y., \& Jiang, Y. V. (2014). Spatial working memory interferes with explicit, but not probabilistic cuing of spatial attention. Journal of Experimental Psychology: Learning, Memory, and Cognition, 41(3), 787-806. doi:10.1037/xlm0000040 